\title{
Reduced experimental autoimmune encephalomyelitis after intranasal and oral administration of recombinant lactobacilli expressing myelin antigens
}

\author{
Catharina B.M. Maassen ${ }^{\mathrm{a}, \mathrm{b}, *}$, Jon D. Laman ${ }^{\mathrm{b}}$, Conny van Holten-Neelen ${ }^{\mathrm{a}}$, \\ Linsy Hoogteijling ${ }^{a}$, Lizet Groenewegen ${ }^{a}$, Lizette Visser ${ }^{b}$, \\ Marc M. Schellekens ${ }^{a}$, Wim J.A. Boersma ${ }^{c}$, Eric Claassen ${ }^{b}$ \\ a Division of Immunological and Infectious Diseases, TNO-Prevention and Health (TNO-PG), \\ P.O. Box 2215, 2301 CE Leiden, The Netherlands \\ ${ }^{\mathrm{b}}$ Department of Immunology, Erasmus University Rotterdam, P.O. Box 1738, 3000 DR Rotterdam, The Netherlands \\ ${ }^{c}$ Animal Sciences Group, Division of Animal Resources Development, P.O. Box 65, 8200 AB Lelystad, The Netherlands
}

Received 20 May 2003; received in revised form 17 June 2003; accepted 30 June 2003

\begin{abstract}
Oral administration of autoantigens is a safe and convenient way to induce peripheral T-cell tolerance in autoimmune diseases like multiple sclerosis (MS). To increase the efficacy of oral tolerance induction and obviate the need for large-scale purification of human myelin proteins, we use genetically modified lactobacilli expressing myelin antigens. A panel of recombinant lactobacilli was constructed producing myelin proteins and peptides, including human and guinea pig myelin basic protein (MBP) and proteolipid protein peptide 139-151 ( PLP $\left._{139-151}\right)$. In this study we examined whether these Lactobacillus recombinants are able to induce oral and intranasal tolerance in an animal model for multiple sclerosis, experimental autoimmune encephalomyelitis (EAE). Lewis rats received soluble cell extracts of Lactobacillus transformants intranasally three times prior to induction of EAE. For the induction of oral tolerance, rats were fed live transformed lactobacilli for 20 days. Ten days after the first oral administration EAE was induced. Intranasal administration of extracts containing guinea pig MBP (gpMBP) or $\mathrm{MBP}_{72-85}$ significantly inhibited EAE in Lewis rats. Extracts of control transformants did not reduce EAE. Live lactobacilli expressing guinea pig $\mathrm{MBP}_{72-85}$ fused to the marker enzyme $\beta$-glucuronidase ( $\beta$-gluc) were also able to significantly reduce disease when administered orally. In conclusion, these experiments provide proof of principle that lactobacilli expressing myelin antigens reduce EAE after mucosal (intranasal and oral) administration. This novel method of mucosal tolerance induction by mucosal administration of recombinant lactobacilli expressing relevant autoantigens could find applications in autoimmune disease in general, such as multiple sclerosis, rheumatoid arthritis and uveitis.
\end{abstract}

(C) 2003 Elsevier Ltd. All rights reserved.

Keywords: Mucosa; Autoimmunity; Tolerance/suppression

\section{Introduction}

Multiple sclerosis (MS) is a chronic inflammatory disease of the central nervous system (CNS) that leads to destruction of CNS myelin. It is widely assumed that MS is an autoimmune disease mediated by $\mathrm{CD} 4+\mathrm{T}$ cells of

Abbreviations: MS, multiple sclerosis; EAE, experimental autoimmune encephalomyelitis; APL, altered peptide ligand; gp, guinea pig; MBP, myelin basic protein; PLP, proteolipid protein peptide; $\beta$-gluc, $\beta$-glucuronidase

* Corresponding author. Present address: Symbigene B.V., Edelhertweg 15, 8219 PH Lelystad, The Netherlands.

Tel.: +31-32-023-8996/71-518-1545; fax: +31-32-023-8094/71-518-1909.

E-mail address: cmaassen@symbigene.com (C.B.M. Maassen). the $\mathrm{T}$ helper 1 subset. The current therapies available for MS are non-specific, may have toxic side effects or cannot be used in long-term treatment. Therefore, antigen-specific therapies to silence or delete autoreactive $\mathrm{T}$ cells are preferred. In animal models for MS (experimental autoimmune encephalomyelitis (EAE)), induction of peripheral T-cell tolerance can be achieved by injection of large doses of soluble myelin antigens intravenously or intrathymically [1,2] (reviewed by Liblau et al. [3]). Also systemic administration of altered peptide ligands (APL) or MHC-II-peptide complexes can suppress EAE in an antigen-specific manner (reviewed by Liblau et al. [3]). For use in humans a disadvantage is that some of these therapies need to be tailored to the HLA haplotype of individual patients. 
A promising antigen-specific therapy is the induction of peripheral T-cell tolerance by mucosal administration of autoantigen (mucosal tolerance). Mucosal tolerance by oral and intranasal administration has been achieved with several different myelin antigens in distinct EAE models (e.g. [4-7]). Three main mechanisms of mucosal T-cell tolerance induction have been described; anergy, deletion and active suppression. When anergy (non-responsiveness of autoreactive $\mathrm{T}$ cells) $[8,9]$ or deletion [10] are the desired mechanisms of peripheral T-cell tolerance induction, the autoantigen needs to be known. This obstacle can be circumvented when active bystander suppression is induced [4,6,11,12]. A local Th2/Th3 (e.g. [13]) environment or a suppressive CD8+ T-cell subset $[14,15]$ can suppress the inflammatory autoimmune response.

In the Lewis rat, EAE can be induced by immunization with guinea pig spinal cord homogenate, myelin basic protein (MBP) or proteolipid protein peptide (PLP) emulsified in complete Freund's adjuvant (CFA). The dominant encephalitogenic epitope of MBP in the Lewis rat is $\mathrm{MBP}_{72-85}$ $[16,17]$. EAE induced with this peptide is mediated by CD4+ T cells. A second MBP epitope encephalitogenic for Lewis rats has been mapped to residues 86-98 [18,19]. However, this peptide is only weakly encephalitogenic, requiring higher doses to elicit clinical signs [20]. Several groups have reported that intranasal administration of autoantigens or peptides derived from self as well as foreign antigens can also induce peripheral T-cell tolerance [7,21-25]. However, to our knowledge, intranasal tolerance induction has not been reported yet in $\mathrm{MBP}_{72-85}$ induced $\mathrm{EAE}$ in Lewis rats.

Mucosal administration of autoantigens is very effective in preventing induction of autoimmune disease in animal models, but in the human situation chronic disease needs to be treated. In some experimental autoimmune models, it is possible to treat ongoing disease $[4,26]$. The efficacy of these treatments is crucially dependent on dosing and scheduling [27]. For several autoimmune diseases in human, therapy by oral administration of antigen has been attempted. Although some clinical trials were promising, no clear positive results were obtained (e.g. [28-30]). For MS, one of the problems is the fact that the autoantigen is still unknown, a problem that does not exist in animal models. Another major problem is the source, the purity and the amount of antigen that needs to be obtained. In the human MS trial, bovine myelin was used. From animal studies it is known that heterogenous antigen mixtures such as myelin are less effective in inducing oral tolerance than single protein antigens such as purified MBP [26]. In addition, very high doses are required in oral tolerance induction, partially due to breakdown of antigen in the stomach. In order to overcome a number of these problems, we used genetically modified lactobacilli that produce the antigen locally in the gut. The use of these recombinant lactobacilli is probably safer than purified human or animal myelin, because there is no risk of administering viruses or prions co-isolated with myelin. A panel of recombinant Lactobacillus strains was constructed which produce myelin proteins and peptides, including human MBP, guinea pig MBP (gpMBP) and PLP ${ }_{139-151}$ [31]. cDNAs of a number of encephalitogenic myelin proteins and peptides were cloned, because encephalitogenic antigens have been demonstrated to be efficient tolerogens (e.g. $[8,22])$.

Lactobacilli are Gram-positive lactic acid bacteria which are frequently used in dairy products because of their health promoting effects such as the non-specific enhancement of the immune response (adjuvanticity), control of intestinal infections, control of serum cholesterol levels and anti-carcinogenic activity [32]. Oral or intranasal administration of these diverse species of lactic acid bacteria with the generally regarded as safe (GRAS) status is cost-effective and simple [33]. Since individual Lactobacillus strains are clearly distinct in various properties, strain selection is very important. We have chosen Lactobacillus casei for recombinant autoantigen expression, because this strain possibly favors tolerance induction in the gut by inducing TGF- $\beta$ and IL-10 expression [34], while the bacterium itself is not immunogenic and does not enhance the humoral immune response to exogenous protein antigen in a non-specific manner [35].

The aim of the current study was to determine whether mucosal administration of recombinant lactobacilli expressing myelin antigens could reduce EAE. Our data show that Lactobacillus recombinants can prevent EAE by oral and intranasal administration, and that in this particular model, intranasal administration of purified gpMBP can also enhance EAE.

\section{Materials and methods}

\subsection{Animals}

Female Lewis rats of approximately $175 \mathrm{~g}$ were obtained from Charles River/The Broekman Institute, Someren, The Netherlands. All animals were kept under filtertop hoods in a DII facility with free access to pelleted food and acidified water (pH 2.8). Experiments were performed according to regulations in the Dutch laws on animal experimentation and on the use of genetically modified microorganisms.

\subsection{Recombinant lactobacilli}

For the induction of tolerance by oral or intranasal administration of recombinant lactobacilli in Lewis rats two groups of vectors were used, the pLP402 series and the pLP403 series (Table 1). All pLP402 vectors secrete heterologous protein, whereas the pLP403 vectors retain the heterologous protein intracellularly. The basic Escherichia coli/Lactobacillus shuttle vectors and the general construction method have been described by Maassen et al. [31]. All pLP400/u vectors express a fusion protein 
Table 1

Recombinant lactobacilli expressing heterologous antigens for the induction of mucosal tolerance ${ }^{\mathrm{a}}$

\begin{tabular}{|c|c|c|}
\hline $\begin{array}{l}\text { Localization of } \\
\text { heterologous } \\
\text { antigen }\end{array}$ & Code vector & Heterologous protein/peptide \\
\hline \multirow[t]{2}{*}{ Secreted } & pLP402-gpMBP & gpMBP \\
\hline & $\begin{array}{l}\mathrm{pLP} 402-\mathrm{gpMBP} / \mathrm{u} \\
\mathrm{pLP} 402-\mathrm{MBP} 72 / \mathrm{u} \\
\mathrm{pLP} 402 / \mathrm{u}\end{array}$ & $\begin{array}{l}\text { gpMBP fused to } \beta \text {-gluc } \\
\text { MBP }_{72-85} \text { fused to } \beta \text {-gluc } \\
\beta \text {-gluc }\end{array}$ \\
\hline Intracellular & pLP403-MBP72/u & $\mathrm{MBP}_{72-85}$ fused to $\beta$-gluc \\
\hline
\end{tabular}

${ }^{a}$ A panel of vectors for the expression of myelin antigens in lactobacilli was constructed. The expression of the heterologous antigens is driven by the regulatable amy promoter. The panel consists of vectors which express peptide and protein antigens, fused to the marker enzyme $\beta$-glucuronidase (/u) or not. All pLP402 vectors secrete heterologous protein, whereas the pLP403 vectors retain the heterologous antigen intracellularly.

with the marker enzyme $\beta$-glucuronidase $(\beta$-gluc) from E. coli (Table 1). Vectors were transformed to L. casei (ATCC 393).

\subsection{Culturing of recombinant lactobacilli}

For oral administration of recombinant lactobacilli, the cells were prepared as described below. One liter of mMRS containing 1\% mannitol and erythromycin [31] was inoculated at 1:200 with a stationary phase culture of the recombinant Lactobacillus strains and cultured without aeration at $37^{\circ} \mathrm{C}$ till an $\mathrm{OD}_{690}$ of 1.0 was reached. For all recombinants used in this study the highest level of heterologous gene expression was approximately at the optical density of 1.0. The cells were harvested and washed twice with PBS and once with $0.2 \mathrm{M} \mathrm{NaHCO}_{3}$. The cells were resuspended in $\mathrm{NaHCO}_{3}$ to a volume of $12 \mathrm{ml}$. A small volume was plated to calculate the number of colony forming units (CFU) orally administered.

For the induction of tolerance by intranasal administration, extracts of recombinant lactobacilli were used. The cells were grown as described above, harvested and washed with PBS. The extracts were made by sonicating the cells in PBS as described before [31]. The soluble fraction was used for intranasal administration.

\subsection{Intranasal tolerance induction}

Rats received either $80 \mu \mathrm{l}$ of Lactobacillus extracts ( $\approx 320 \mu \mathrm{g}$ total protein), synthetic $\mathrm{MBP}_{72-85}$ (QKSQRSQDENPV) (100 or $200 \mu \mathrm{g})$, purified gpMBP [36] (100 or $200 \mu \mathrm{g}$ ) or PBS divided over two nostrils (Table 1). Intranasal administration of cell extracts of lactobacilli containing heterologous antigens took place at days -15 , -10 and -5 . All vectors and their relevant characteristics used for induction of tolerance have been summarized in Table 1. At day 0, EAE was induced with $\mathrm{MBP}_{72-85}$ [37].

\subsection{Oral tolerance induction}

Rats intragastrically received cells of one strain of recombinant lactobacilli or $\mathrm{NaHCO}_{3}$ buffer as a control, daily for 20 days (from days -10 to 9 ). The cells were prepared as described above. The rats received approximately $2 \times 10^{11}$ lactobacilli in $2 \mathrm{ml}$ with a gastric syringe. At day 0 , EAE was induced with $\mathrm{MBP}_{72-85}$.

\subsection{EAE induction in Lewis rats}

EAE was induced by s.c. immunization in the hind footpads with a total of $70 \mu \mathrm{g} \mathrm{MBP}_{72-85}$ (Table 1) emulsified in Difco's incomplete adjuvant with $4 \mathrm{mg} / \mathrm{ml}$ Mycobacterium tuberculosis H37Ra (Difco, Detroit, MI). Clinical disease was monitored daily from day 6 onward by weighing the rats, and by grading symptoms of paralysis using an internationally accepted clinical scoring scale ranging from 0 (no signs) to 5 (death) due to EAE (see [38]). In Figs. 1 and 2, the term cumulative EAE score has been used as a measure for the severity of the disease. It was calculated by adding up all the scores per animal over the first 21 days after EAE induction, in other words, calculating the complete area under the curve, representing the total disease load. The cumulative EAE score of each animal was calculated as a percentage of the mean cumulative EAE score of the control group within the same experiment, which was set at $100 \%$. Because every animal was compared to its own

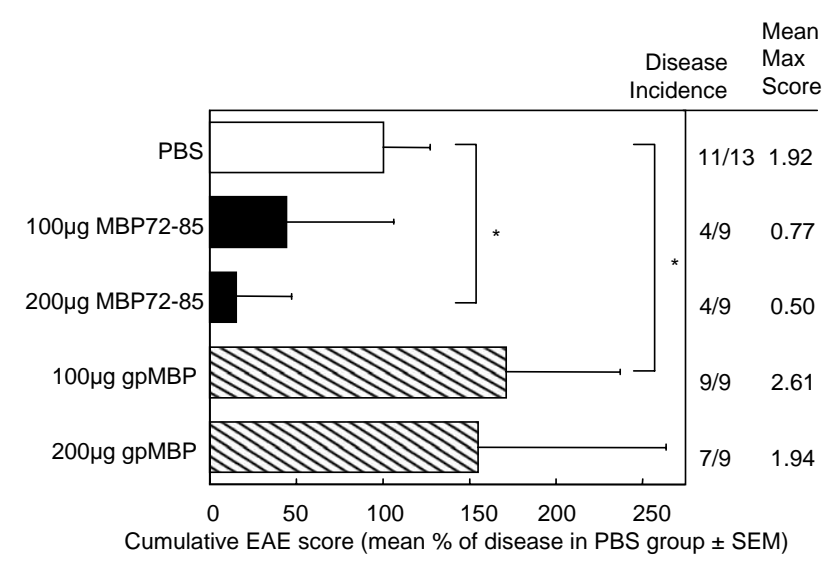

Fig. 1. Exacerbation versus inhibition of EAE in Lewis rats by intranasal administration of MBP protein or peptide. At days $-15,-10$ and -5 Lewis rats intranasally received 100 or $200 \mu \mathrm{g}$ MBP peptide $72-85$ (filled bars) or gpMBP protein (slashed bars). At day 0, EAE was induced with $\mathrm{MBP}_{72-85}$. The cumulative EAE score of each animal was determined by adding up all EAE scores and was expressed as a percentage of the mean cumulative EAE score of the control group which received intranasally PBS only within the same experiment. The cumulative EAE score per treatment over three experiments was determined by calculating the mean cumulative EAE score of all animals which had received the same treatment. Disease incidence indicates the number of animals which had a score of 0.5 or higher related to the number of animals per treatment. The mean max score is the mean of the highest score of each animal during the disease course. ${ }^{*} P<0.05$ compared to control group which nasally received PBS (open bars). 


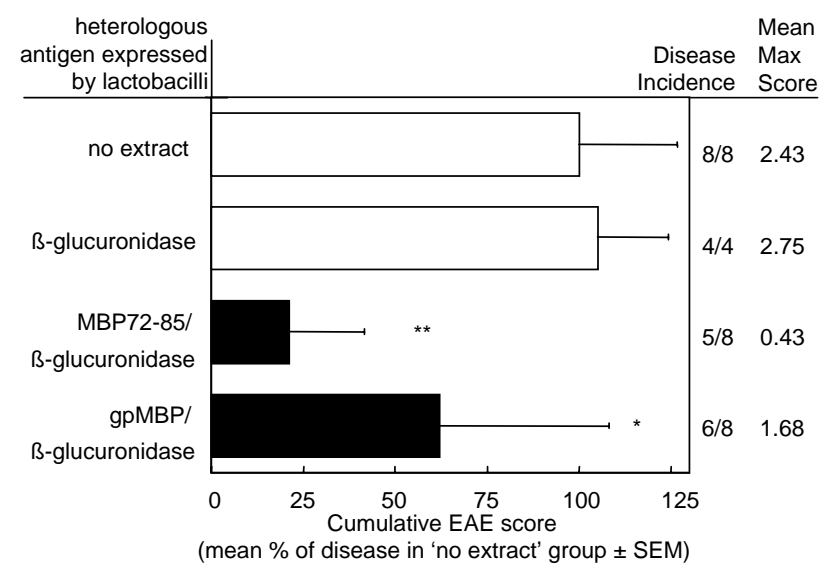

Fig. 2. Reduction of EAE after intranasal administration of Lactobacillus extracts containing myelin antigens in Lewis rats. At days $-15,-10$ and -5 , Lewis rats intranasally received $80 \mu \mathrm{l}$ Lactobacillus extract containing approximately $320 \mu \mathrm{g}$ protein. The Lactobacillus contained expressed heterologous myelin antigen fused to $\beta$-gluc (filled bars) or $\beta$-gluc only (open bars). At day 0, EAE was induced with $\mathrm{MBP}_{72-85}$. The cumulative EAE score was calculated as in Fig. 1 and expressed as a percentage of the mean cumulative EAE score of the control group which intranasally received PBS only. The results of two experiments are shown. For disease incidence and mean max score, see legend Fig. $1 .{ }^{*} P<0.05$ compared to both control groups (open bars), ${ }^{* *} P<0.01$ compared to both control groups (open bars).

positive control, we were able to combine experiments in spite of small variations between the control groups.

\subsection{ELISA}

Serum was collected every 7 days and tested in ELISA for the presence of antibodies against myelin proteins and peptides as a measure of T- and B-cell stimulation and tolerance induction. Plates were coated with $5 \mu \mathrm{g} / \mathrm{ml}$ antigen in PBS $\left(50 \mu l\right.$ per well) overnight at $4{ }^{\circ} \mathrm{C}$. Non-specific antibody binding was blocked by incubation with $0.2 \%$ gelatin in PBS $\left(50 \mu \mathrm{l}\right.$ per well) for $1 \mathrm{~h}$ at $25^{\circ} \mathrm{C}$. Subsequently the plates were incubated with dilutions of serum of treated animals and preimmune sera to correct for background reactivity for $1 \mathrm{~h}$ at room temperature. For the detection of serum IgG antibodies specific for the diverse myelin antigens in rat, alkaline phosphatase-labeled goat anti-rat $\operatorname{IgG}$ (Sigma Chemical Co., La Jolla, CA) was used. At several time points after addition of the substrate paranitrophenyl phosphate, the absorbance was read at $405 \mathrm{~nm}$.

\subsection{Immunoblot analysis}

Proteins in cell extracts were separated by SDS-polyacrylamide gel electrophoresis (PAGE) $(7.5,10$ or $14 \%$ acrylamide, $400 \mathrm{mM}$ Tris $\mathrm{pH} 8.8$ ) and run in a $25 \mathrm{mM}$ Tris, $192 \mathrm{mM}$ glycine buffer ( $\mathrm{pH} 8.3$ ) at $200 \mathrm{~V}$ for $45 \mathrm{~min}$. Protein was transferred electrophoretically onto nitrocellulose using a Bio-Rad Miniprotean II blotting unit (Bio-Rad
Laboratories, Hercules, CA). Immunoblots were incubated with optimally diluted rabbit anti-gpMBP or rabbit anti- $\beta$-glucuronidase antiserum. Antibodies against gpMBP and $\beta$-glucuronidase were induced by immunization of New Zealand White rabbits with gpMBP and $\beta$-glucuronidase in complete adjuvant containing $M$. tuberculosis $H 37 R a$ (Difco Laboratories). The second incubation step was performed with swine anti-rabbit Ig-HRP (Dako A/S, Glostrup, Denmark). The immunoblots were incubated with ECL detection reagents (Amersham Life Science Ltd., Buckinghamshire, UK). A light sensitive film (Kodak X-omat) was exposed to the blots for a variety of time periods before development. In all immunoblots, guinea pig MBP or $\beta$-glucuronidase (Sigma Chemical Co.) were used as references for the immuno-reagents.

The amount of soluble heterologous proteins was calculated by comparison of the relative intensity of bands of several dilutions of extracts of transformants with a reference of purified $\beta$-glucuronidase or purified gpMBP in immunoblot analysis making use of Bio-1D V6.32 Software (Vilber Lourmat, Marne la Vallée, France).

\subsection{Statistics}

The term cumulative EAE score is used as measure for the severity of the disease. It was calculated by adding up all the scores per animal over the first 21 days after EAE induction. In other words, the cumulative EAE score is the total area under the curve. The cumulative EAE score of each animal was calculated in percentages of the mean cumulative EAE score of the control group which received PBS intranasally or $\mathrm{NaHCO}_{3}$ orally only within the same experiment. The cumulative EAE score per treatment over several experiments was determined by calculating the mean cumulative EAE score of all animals that had received the same treatment and is expressed as a percentage of the control group which is set at $100 \%$. In order to determine whether differences between groups reached significance, statistical analysis was performed using a single factor ANOVA, followed by calculating the least significant difference.

\section{Results}

\subsection{Exacerbation versus inhibition of EAE by intranasal administration of MBP protein or peptide}

To investigate whether it was possible to induce tolerance in Lewis rats by intranasal administration of myelin antigen, gpMBP and the immunodominant synthetic peptide $\mathrm{MBP}_{72-85}$ were administered intranasally before induction of EAE. The protein and the peptide were administered in two doses of 100 or $200 \mu \mathrm{g}$ at 15,10 and 5 days before induction of EAE with $\mathrm{MBP}_{72-85}$ (Fig. 1). Intranasal administration of the MBP peptide 72-85 partially ameliorated EAE in a dose dependent manner. When $100 \mu \mathrm{g}$ peptide 
was administered per application, the cumulative EAE score was reduced with $56 \%$. A significant reduction of EAE with $85 \%$ compared to the control group was found when $200 \mu \mathrm{g}$ $\mathrm{MBP}_{72-85}$ was given intranasally $(P<0.05)$. This is the first time that mucosal tolerance induction was demonstrated in this EAE model using $\mathrm{MBP}_{72-85}$ as disease inducing antigen in rats. In contrast, when the whole MBP protein was administered intranasally, an enhancement of EAE was found. The increase of the cumulative EAE score was comparable for both doses applied $(100 \mu \mathrm{g}=171 \%, 200 \mu \mathrm{g}=155 \%)$, and this was significant for the $100 \mu \mathrm{g}$ dose $(P<0.05)$.

\subsection{Intranasal tolerance induction by Lactobacillus extracts containing myelin antigens}

Prior to intranasal application of the soluble fraction of recombinant lactobacilli extracts containing myelin antigens for tolerance induction, proper expression of myelin antigens by the recombinants was analyzed. The Lactobacillus recombinants containing the constructs pLP402-MBP72/u and pLP402-gpMBP/u secrete $\mathrm{MBP}_{72-85}$ peptide and gpMBP protein fused to the marker enzyme $\beta$-gluc, respectively. The Lactobacillus recombinant containing the construct pLP402/u, which secretes $\beta$-gluc only, was used as a negative control (Table 1).

Expression of these heterologous proteins by L. case $i$ was confirmed by immunoblotting with anti- $\beta$-glucuronidase antibody ([31], results not shown). The heterologous protein gpMBP fused to $\beta$-gluc was also detected with anti-gpMBP antibody [31]. The expression of fusion protein $\mathrm{MBP}_{72-85} / \beta$-gluc was also demonstrated with anti-MBP $72-85$ antibody (results not shown). The soluble fraction of extracts of recombinant lactobacilli was administered intranasally three times, at days $-15,-10$ and -5 prior to EAE induction with $\mathrm{MBP}_{72-85}$ on day 0 (Fig. 2). Intranasal application of Lactobacillus extracts of the control recombinant pLP402/u did not affect the EAE course, as expected. Intranasal pretreatment with Lactobacillus extracts of $\mathrm{pLP} 402$-gpMBP/u reduced the mean cumulative EAE score significantly (about 40\%) when compared to either of both control groups (PBS and lactobacilli expressing $\beta$-gluc only) (both $P<0.05$ ). When Lactobacillus extracts containing $\mathrm{MBP}_{72-85}$ were applied intranasally, EAE was even further reduced. In this case, the mean cumulative EAE score was reduced with almost $80 \%(P<0.01)$ compared to the PBS treated group (Fig. 2).

\subsection{Oral tolerance induction by recombinant lactobacilli expressing $M B P_{72-85}$ intracellularly or extracellularly}

Three recombinant Lactobacillus strains were used to test whether oral administration of live lactobacilli expressing myelin antigens could prevent EAE. The strains previously used in the intranasal tolerance induction experiments, pLP402-MBP72/u (secretion of $\mathrm{MBP}_{72-85}$ fused to $\beta$-gluc) and the control strain pLP402/u (secretion of $\beta$-gluc), were used again. In addition, the strains with pLP402-gpMBP, which secretes gpMBP protein and pLP403-MBP72/u, which intracellularly retains the $\mathrm{MBP}_{72-85}$ peptide fused to $\beta$-gluc, were used (Table 1 ). Approximately $2 \times 10^{11}$ cells were orally administered per animal daily from days -10 to 10 . At day 0 , rats were immunized with $\mathrm{MBP}_{72-85}$ to induce EAE. When lactobacilli expressing $\beta$-gluc only (pLP402/u) were administered orally, only three out of five rats developed EAE, but no significant difference was found in day of onset, mean maximum score or mean cumulative EAE score, when compared to the control group who had received buffer $\left(\mathrm{NaHCO}_{3}\right)$ orally (disease incidence 100\%) (Fig. 3). Oral administration of lactobacilli secreting gpMBP (pLP402-gpMBP) had no effect on EAE. However, EAE was significantly inhibited when lactobacilli secreting the MBP peptide 72-85 (pLP402-MBP72/u) was administered (Fig. 3). This treatment reduced the mean cumulative EAE score with $65 \%$ compared to the $\mathrm{NaHCO}_{3}$
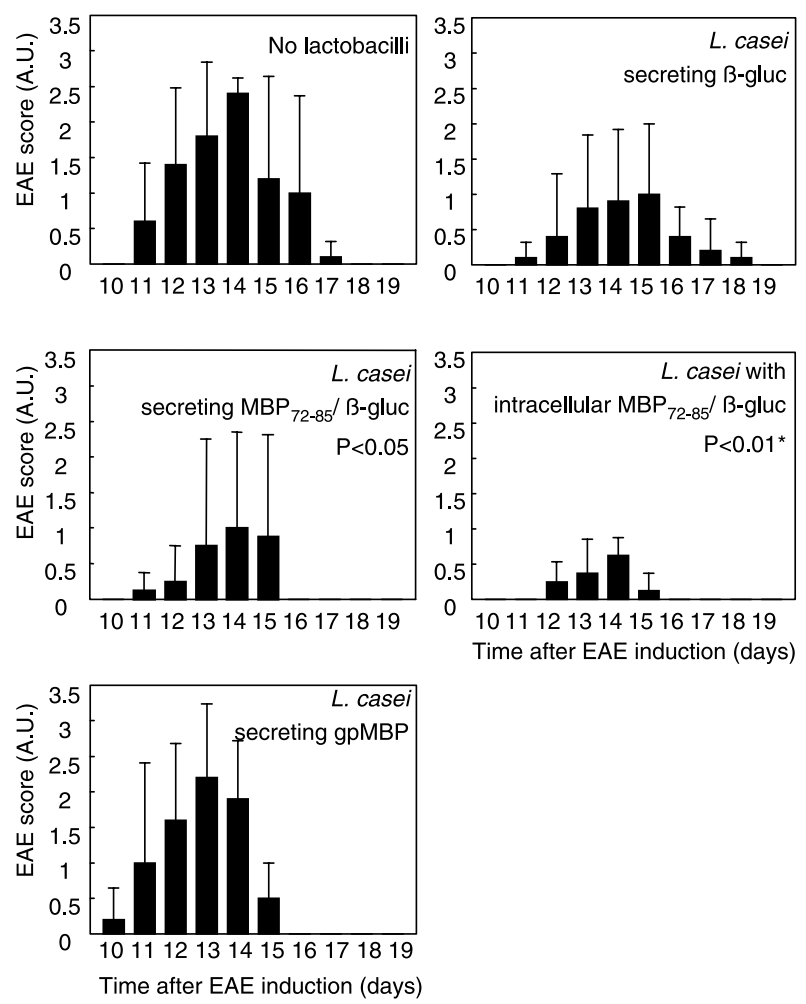

Fig. 3. Oral tolerance induction in Lewis rats by recombinant lactobacilli expressing $\mathrm{MBP}_{72-85}$ intracellularly or extracellularly. Groups of five rats received orally $2 \times 10^{11}$ live recombinant lactobacilli expressing myelin antigens for 20 days (days -10 to 10 ). The vectors used were designed for intracellular expression or secretion of the heterologous antigen. EAE was induced with $\mathrm{MBP}_{72-85}$ at day 0 . The mean EAE score in arbitrary units per experimental group is shown as a function of time after EAE induction. The cumulative EAE score (area under the curve) was calculated as in Fig. 1 and used for statistical analysis. The $P$-values shown represent the $P$-value of the statistical analysis of the relevant experimental group compared to the control group which received no lactobacilli orally. (*) means the group which received $L$. casei expressing $\mathrm{MBP}_{72-85}$ intracellular, also was significantly different from the group which received $L$. casei expressing gpMBP $(P<0.05)$. 
buffer control group $(P<0.05)$. An even further reduction was achieved by oral administration of lactobacilli recombinants with retain the $\mathrm{MBP}_{72-85}$ peptide intracellularly (pLP403-MBP72/u). The mean cumulative EAE score was reduced by $84 \%$ compared to the buffer control group $(P<0.01)$. This reduction was also significant compared to the group orally treated with pLP402-gpMBP $(P<0.05)$ (Fig. 3).

\subsection{Antibody responses after intranasal tolerance induction}

In all rat experiments the animals were immunized with $\mathrm{MBP}_{72-85}$ to induce EAE. In none of the animals an anti-MBP ${ }_{72-85}$ antibody response could be detected, despite the fact that T-cell priming was effective as evidenced by a $100 \%$ disease incidence in control groups. In animals not treated for tolerance induction which were immunized with $\mathrm{MBP}_{72-85}$, no $\mathrm{MBP}$ specific antibodies could be detected. This gave us the opportunity to investigate whether intranasal application of gpMBP-induced gpMBP-specific systemic antibodies. Only in animals with an enhanced cumulative EAE score after intranasal treatment with gpMBP, IgG antibodies specific for gpMBP could be detected in serum 14 days after EAE induction. This implies that intranasal administration of gpMBP induces a specific antibody response. No gpMBP-specific antibody responses could be detected in any of the rats which mucosally received lactobacilli expressing MBP or MBP peptide.

\section{Discussion}

\subsection{Novel findings of this study}

There have been several studies in which recombinant lactobacilli have shown their promise as a vaccine carrier [39-42] and as a candidate therapeutic for the treatment of allergic disorders [43]. This study is the first demonstration of interference in EAE, a Th1-cell driven autoimmune disease of the central nervous system by mucosally administered recombinant lactobacilli producing relevant myelin autoantigens. In this study, we have demonstrated that intranasal as well as oral administration of recombinant lactobacilli expressing myelin antigens could effectively reduce EAE. In this rat model where EAE was induced by $\mathrm{MBP}_{72-85}$, mucosal tolerance induction has not been demonstrated before. Several other groups have shown that mucosal administration of an autoantigen can enhance clinical signs $[27,44,45]$. Here, we show in a different model, nasal administration of purified gpMBP in $\mathrm{MBP}_{72-85}$ induced EAE, that enhancement of disease can also occur. In EAE, such augmentation of disease only has been seen with MBP isolated from spinal cord, which could suggest that impurities, such as other encephalitogenic myelin proteins, are the cause of this effect [46]. The use of recombinant proteins could prevent this problem. As demonstrated in this study, expression of such proteins/peptides by lactobacilli might even further reduce clinical signs and provide a therapy at significantly reduced cost when compared to oral administration of synthetic or purified compounds.

\section{2. gpMBP is less effective than $M B P_{72-85}$ in reducing $M B P_{72-85}$ induced $E A E$}

Intranasal administration of gpMBP (fused to $\beta$-gluc) expressed by lactobacilli did reduce EAE significantly, but less effectively than when lactobacilli expressing $\mathrm{MBP}_{72-85}$ fused to $\beta$-gluc were used. Highly similar results were obtained by oral administration of lactobacilli expressing gpMBP or $\mathrm{MBP}_{72-85}$, although in those experiments gpMBP was not fused to $\beta$-gluc. In contrast to the experiments performed with purified gpMBP and synthetic peptide (Fig. 1), approximately equimolar amounts of recombinant gpMBP and $\mathrm{MBP}_{72-85}$ both fused to $\beta$-gluc were administered (Fig. 3). This was deduced from immunoblots that showed roughly equal amounts of $\beta$-gluc per unit weight of total Lactobacillus protein. Consequently, the same is true for the heterologous antigen. This was the case for all secretory vectors. Therefore, the difference in inhibition of EAE between lactobacilli with secretory vectors cannot be explained by a difference in the molar number of epitopes delivered. Conformational differences between recombinant $\mathrm{MBP}_{72-85}$ and gpMBP both fused to $\beta$-gluc may affect intracellular proteolytic processing, and subsequently T-cell reactivity. Differences in susceptibility to extracellular proteases can also play a role.

\subsection{Presentation of recombinant myelin antigens to the mucosal immune system}

According to the general dogma, mucosal administration of soluble antigens leads to systemic T-cell tolerance whereas particulate antigens can induce local and systemic humoral and cellular responses (e.g. [26,47]). Therefore, we expected that oral administration of lactobacilli secreting soluble antigens would be more effective in reducing EAE than lactobacilli that retain the antigen intracellularly. Contrary to this expectation, lactobacilli that expressed $\mathrm{MBP}_{72-85}$ fused to the marker enzyme $\beta$-gluc exclusively intracellularly appeared to reduce EAE more effectively than lactobacilli secreting the peptide-fusion protein. This could simply be the result of the higher expression level of the intracellularly expressed heterologous protein (approximately 3 times higher than secretory $\mathrm{MBP}_{72-85}$ fused to $\beta$-gluc). Interpretation of these findings is hampered by the general lack of insight into behavior of Lactobacillus strains in the gut, as well as cellular uptake and processing of lactobacilli and their intracellular or secreted antigens. 


\subsection{Lactobacilli promote tolerance induction}

In the intranasal administration experiments lower doses of recombinant antigen were able to prevent EAE than those required to prevent EAE with synthetic peptide $(100 \mu \mathrm{g})$ (Fig. 1). Significant reduction of EAE was demonstrated with Lactobacillus extracts containing approximately $1 \mu \mathrm{g}$ $\mathrm{MBP}_{72-85}$ (Fig. 2). Application of lactobacilli extracts containing $\sim 10 \mu \mathrm{g}$ gpMBP showed reduction of EAE, whereas $100 \mu \mathrm{g}$ purified MBP augmented the clinical signs (Fig. 1 versus Fig. 2). This indicates that the presence of Lactobacillus antigens might have further reduced EAE, even though administration of only lactobacilli in itself did not have any effect on the disease course (Fig. 2, lactobacilli expressing $\beta$-gluc). However, when lactobacilli expressing $\beta$-gluc were administered orally, we did observe a reduction of EAE although this was not significant. That this is due to the administration of the lactobacilli by itself is unlikely, because in a previous study we demonstrated that wild type administration of $L$. casei did not influence EAE disease course [48]. In addition, also lactobacilli expressing the heterologous protein MBP can be regarded as vector control, because these recombinant lactobacilli did not effect EAE disease course either. Although an effect of $\beta$-gluc on EAE is very unlikely it cannot be excluded, in spite of the fact that it has never been seen in nasal rat experiments nor in nasal and oral mouse EAE experiments (data not shown).

Our data might suggest that lactobacilli may have additional beneficial effects also in the oral tolerance experiments on the reduction of EAE. Lewis rats which were fed lactobacilli containing approximately $25 \mu \mathrm{g} \mathrm{MBP}_{72-85}$ for 20 days showed significantly reduced signs of EAE. The cumulative amount of $\mathrm{MBP}_{72-85}$ peptide was still 10-fold lower than the doses used to suppress $\mathrm{MBP}_{68-88}$ induced EAE with $\mathrm{MBP}_{68-88}$ (four times $1.25 \mathrm{mg}$ peptide) [49]. However, this claim requires additional experiments with mg doses of peptide. If confirmed, such effects of lactobacilli could be based on the protection of protein by the lactobacilli against degradation, and the particulate nature of lactobacilli versus the soluble nature of peptides.

\subsection{Mucosal administration of low antigen doses can enhance disease in rats}

Comparable doses of $\mathrm{MBP}_{72-85}$ peptide reduced $\mathrm{MBP}_{72-85}$ induced EAE to the same degree as was demonstrated in gpMBP-induced EAE [22]. In contrast, EAE induced by $\mathrm{MBP}_{72-85}$ was enhanced by intranasal administration of gpMBP, although it has been demonstrated that it is possible to induce oral tolerance with intact protein (MBP and PLP) in peptide (PLP $\left.{ }_{140-159}\right)$ induced EAE [11].

It is known that the dose and administration regimen is crucial for the induction of mucosal tolerance. Oral administration of low doses can enhance disease, as was demonstrated by Meyer et al. [27]. Also feeding of very low doses of OVA appears to prime rather than tolerize the immune response, resulting in enhanced delayed type hypersensitivity responses [50]. Based on molarity, an approximately 15-fold lower number of the $\mathrm{MBP}_{72-85}$ epitope was present in intranasally administered gpMBP, as compared to the synthetic $\mathrm{MBP}_{72-85}$ peptide administered. In our experiments the amount of intranasally administered gpMBP equaled 6.7 and $13.3 \mu \mathrm{g} \mathrm{MBP}_{72-85}$ peptide, indicating that much lower doses were administered than the $100 \mu \mathrm{g}$ synthetic $\mathrm{MBP}_{72-85}$ peptide which not even completely prevented EAE induction after intranasal administration. However, this dose-related explanation is not consistent with the findings that low doses (five times $6 \mu \mathrm{g}$ gpMBP) can prevent disease in a different EAE model (induced with gpMBP) in the Lewis rat [7]. Possibly, the fact that gpMBP contains more T- and B-cell epitopes influences its tolerizing properties. Also other myelin components which were retained in the purified MBP fraction could have affected the immune response. Benson et al. [26] have demonstrated that a heterogenous antigen preparation such as myelin is less effective in inducing tolerance than single antigens (e.g. MBP).

\subsection{Intranasal administration of gpMBP-induced gpMBP-specific antibody responses in rats}

Intranasal administration of gpMBP resulted in enhanced cumulative EAE score of Lewis rats subsequently immunized with $\mathrm{MBP}_{72-85}$ for the induction of EAE. IgG antibody responses were determined as a reflection of $\mathrm{T}$ - and B-cell reactivity. Only in animals with an enhanced cumulative EAE score, MBP specific antibodies could be detected. However, no peptide specific antibody response could be detected in any of the rats after s.c. immunization with $\mathrm{MBP}_{72-85}$, which contains a T-cell epitope. This is in accordance with reports that collectively indicate that $\mathrm{MBP}_{72-85}$ does not contain a complete B-cell epitope for the Lewis rat $[20,51,52]$. Consequently, the MBP specific antibody response detected after intranasal administration of whole gpMBP and s.c. immunization with $\mathrm{MBP}_{72-85}$ is probably only due to the intranasally applied MBP. This indicates that gpMBP-specific antibodies induced by intranasally administered gpMBP correlate with enhancement of EAE.

\subsection{Concluding remarks}

This study provided proof of principle that EAE can be reduced by intranasal as well as oral administration of recombinant lactobacilli expressing myelin antigens. Efficacy of this novel approach may be further improved by optimizing antigen expression levels, bacterial dosing and timing of mucosal administration. The crucial importance of proper dosing and antigen choice in mucosal tolerance induction is emphasized by the enhancement of EAE which we observed upon intranasal administration of gpMBP in Lewis rats. Although the mechanisms of peripheral T-cell tolerance induced by mucosal administration of Lactobacillus recombinants remain to be elucidated, there is accumulating 
evidence that, dependent on the strain used, lactobacilli are able to modulate the immune response in distinct fashions (e.g. $[32,34,35,42,53])$. For instance, in a previous study we have shown that wild type Lactobacillus strains administered orally differentially affect cytokine profiles in the gut. This finding has been further extended by Christensen et al., who have shown that lactobacilli can differentially induce cytokines and MHC surface markers in dendritic cells in vitro [54]. A new model of DC maturation and function was recently proposed, in which immature DC induce T-cell anergy by means of their low MHC, low costimulation and low cytokine production. These authors define a new population of semi-mature DC which develop upon stimulation by factors such as $\mathrm{TNF} \alpha$ and intranasally applied ovalbumin. These semi-mature DC are claimed to suppress immune responses indirectly, by inducing CD4+ regulatory $\mathrm{T}$ cells which produce IL-10, by virtue of their high MHC, high costimulation, but low IL-12, IL-6 and TNF $\alpha$ production. Mature DC induce T-cell immunity through high MHC-peptide, costimulation, and cytokine production [55]. Lutz and Schuler [55] suggest that lactobacilli of the gut flora are one of the signals that induce the semi-mature state of DCs based on the study of Christensen et al. [54]. We previously showed that the L. casei strain used in the current study, induced IL-10 in the gut upon oral administration, as well as low $\mathrm{TNF} \alpha$ and low IL-1 $\beta$. In the same experiment, mice were intraperitoneally immunized with a T-cell dependent antigen. $L$. casei did not enhance the specific antibody response against this antigen, but did induce a high $\mathrm{IgG} / \mathrm{IgG} 2$ a ratio which could be a reflection of a Th2 response. Although very speculative, according to the proposed DC maturation scheme [55], L. casei indeed might be a strain inducing regulatory $\mathrm{T}$ cells as mechanism of tolerance. Independent of the mechanism of action, this study shows that recombinant lactobacilli expressing autoantigens may be suitable as mucosal therapeutic in autoimmune disease in general, such as multiple sclerosis, rheumatoid arthritis and uveitis.

\section{Acknowledgements}

This work was financially supported by grant number MS93-147 from The Netherlands Foundation for the support of Multiple Sclerosis Research.

\section{References}

[1] Goss JA, Nakafusa Y, Roland CR, Hickey WF, Flye MW. Immunological tolerance to a defined myelin basic protein antigen administered intrathymically. J Immunol 1994;153(9):3890-8.

[2] Khoury SJ, Sayegh MH, Hancock WW, Gallon L, Carpenter $\mathrm{CB}$, Weiner HL. Acquired tolerance to experimental autoimmune encephalomyelitis by intrathymic injection of myelin basic protein or its major encephalitogenic peptide. J Exp Med 1993;178(2):559-66.

[3] Liblau R, Tisch R, Bercovici N, McDevitt HO. Systemic antigen in the treatment of T-cell-mediated autoimmune diseases. Immunol Today 1997;18(12):599-604.
[4] Anderton SM, Wraith DC. Hierarchy in the ability of T cell epitopes to induce peripheral tolerance to antigens from myelin. Eur $\mathrm{J}$ Immunol 1998;28(4):1251-61.

[5] Bitar DM, Whitacre CC. Suppression of experimental autoimmune encephalomyelitis by the oral administration of myelin basic protein. Cell Immunol 1988;112(2):364-70.

[6] Higgins PJ, Weiner HL. Suppression of experimental autoimmune encephalomyelitis by oral administration of myelin basic protein and its fragments. J Immunol 1988;140(2):440-5.

[7] Li HL, Liu JQ, Bai XF, van der Meide PH, Link H. Dose-dependent mechanisms relate to nasal tolerance induction and protection against experimental autoimmune encephalomyelitis in Lewis rats. Immunology 1998;94(3):431-7.

[8] Karpus WJ, Kennedy KJ, Smith WS, Miller SD. Inhibition of relapsing experimental autoimmune encephalomyelitis in SJL mice by feeding the immunodominant PLP $\mathrm{P}_{139-151}$ peptide. J Neurosci Res 1996;45(4):410-23.

[9] Whitacre CC, Gienapp IE, Orosz CG, Bitar DM. Oral tolerance in experimental autoimmune encephalomyelitis. III. Evidence for clonal anergy. J Immunol 1991;147(7):2155-63.

[10] Chen Y, Inobe J, Marks R, Gonnella P, Kuchroo VK, Weiner HL. Peripheral deletion of antigen-reactive $\mathrm{T}$ cells in oral tolerance. Nature 1995;376(6536): 177-80.

[11] al-Sabbagh A, Miller A, Santos LM, Weiner HL. Antigen-driven tissue-specific suppression following oral tolerance: orally administered myelin basic protein suppresses proteolipid proteininduced experimental autoimmune encephalomyelitis in the SJL mouse. Eur J Immunol 1994;24(9):2104-9.

[12] Miller A, Lider O, Weiner HL. Antigen-driven bystander suppression after oral administration of antigens. J Exp Med 1991;174(4):791-8.

[13] Chen Y, Kuchroo VK, Inobe J, Hafler DA, Weiner HL. Regulatory $\mathrm{T}$ cell clones induced by oral tolerance: suppression of autoimmune encephalomyelitis. Science 1994;265(5176):1237-40.

[14] Lider O, Santos LM, Lee CS, Higgins PJ, Weiner HL. Suppression of experimental autoimmune encephalomyelitis by oral administration of myelin basic protein. II. Suppression of disease and in vitro immune responses is mediated by antigen-specific CD8+ T lymphocytes. J Immunol 1989;142(3):748-52.

[15] Chen Y, Inobe J, Weiner HL. Induction of oral tolerance to myelin basic protein in CD8-depleted mice: both CD4+ and CD8+ cells mediate active suppression. J Immunol 1995;155(2):910-6.

[16] Hashim GA. Experimental allergic encephalomyelitis in Lewis rats: chemical synthesis of disease-inducing determinant. Science 1977;196(4295):1219-21.

[17] Mannie MD, Paterson PY, Prichard DC, Flouret G. Induction of experimental allergic encephalomyelitis in Lewis rats with purified synthetic peptides: delineation of antigenic determinants for encephalitogenicity, in vitro activation of cellular transfer, and proliferation of lymphocytes. Proc Natl Acad Sci USA 1985;82(16):5515-9.

[18] Offner H, Hashim GA, Celnik B, Galang A, Li XB, Burns FR, et al. $T$ cell determinants of myelin basic protein include a unique encephalitogenic I-E-restricted epitope for Lewis rats. J Exp Med 1989;170(2):355-67.

[19] Sun D, Gold DP, Smith L, Brostoff S, Coleclough C. Characterization of rat encephalitogenic $\mathrm{T}$ cells bearing non- $\mathrm{V}$ beta $8 \mathrm{~T}$ cell receptors. Eur J Immunol 1992;22(2):591-4.

[20] Gould KE, Swanborg RH. T and B cell responses to myelin basic protein and encephalitogenic epitopes. J Neuroimmunol 1993;46(12):193-8.

[21] Dick AD, Cheng YF, Liversidge J, Forrester JV. Intranasal administration of retinal antigens suppresses retinal antigen-induced experimental autoimmune uveoretinitis. Immunology 1994;82(4): $625-31$.

[22] Liu JQ, Bai XF, Shi FD, Xiao BG, Li HL, Levi M, et al. Inhibition of experimental autoimmune encephalomyelitis in Lewis rats by nasal administration of encephalitogenic MBP peptides: synergistic effects of $\mathrm{MBP}_{68-86}$ and $\mathrm{MBP}_{87-99}$. Int Immunol 1998;10(8):1139-48. 
[23] Metzler B, Wraith DC. Inhibition of experimental autoimmune encephalomyelitis by inhalation but not oral administration of the encephalitogenic peptide: influence of MHC binding affinity. Int Immunol 1993;5(9):1159-65.

[24] Staines NA, Harper N, Ward FJ, Malmstrom V, Holmdahl R, Bansal $\mathrm{S}$. Mucosal tolerance and suppression of collagen-induced arthritis (CIA) induced by nasal inhalation of synthetic peptide 184-198 of bovine type II collagen (CII) expressing a dominant $\mathrm{T}$ cell epitope. Clin Exp Immunol 1996;103(3):368-75.

[25] Tian J, Atkinson MA, Clare-Salzler M, Herschenfeld A, Forsthuber T, Lehmann PV, et al. Nasal administration of glutamate decarboxylase (GAD65) peptides induces Th2 responses and prevents murine insulin-dependent diabetes. J Exp Med 1996;183(4):1561-7.

[26] Benson JM, Stuckman SS, Cox KL, Wardrop RM, Gienapp IE, Cross $\mathrm{AH}$, et al. Oral administration of myelin basic protein is superior to myelin in suppressing established relapsing experimental autoimmune encephalomyelitis. J Immunol 1999;162(10):6247-54.

[27] Meyer AL, Benson JM, Gienapp IE, Cox KL, Whitacre CC. Suppression of murine chronic relapsing experimental autoimmune encephalomyelitis by the oral administration of myelin basic protein. J Immunol 1996;157(9):4230-8.

[28] Barnett ML, Kremer JM, St Clair EW, Clegg DO, Furst D, Weisman $\mathrm{M}$, et al. Treatment of rheumatoid arthritis with oral type II collagen. Results of a multicenter, double-blind, placebo-controlled trial. Arthritis Rheum 1998;41(2):290-7.

[29] Weiner HL, Mackin GA, Matsui M, Orav EJ, Khoury SJ, Dawson $\mathrm{DM}$, et al. Double-blind pilot trial of oral tolerization with myelin antigens in multiple sclerosis. Science 1993;259(5099): $1321-4$.

[30] Nussenblatt RB, Gery I, Weiner HL, Ferris FL, Shiloach J, Remaley $\mathrm{N}$, et al. Treatment of uveitis by oral administration of retinal antigens: results of a phase I/II randomized masked trial. Am J Ophthalmol 1997;123(5):583-92.

[31] Maassen CBM, Laman JD, den Bak-Glashouwer MJ, Tielen FJ, van Holten-Neelen JCPA, Hoogteijling L, et al. Instruments for oral disease-intervention strategies: recombinant Lactobacillus casei expressing tetanus toxin fragment $\mathrm{C}$ for vaccination or myelin proteins for oral tolerance induction in multiple sclerosis. Vaccine 1999;17(17):2117-28.

[32] Perdigon G, Alvarez S, Rachid M, Aguero G, Gobbato N. Immune system stimulation by probiotics. J Dairy Sci 1995;78:1597-606.

[33] Pouwels PH, Leer RJ, Boersma WJ. The potential of Lactobacillus as a carrier for oral immunization: development and preliminary characterization of vector systems for targeted delivery of antigens. J Biotechnol 1996;44(1-3):183-92.

[34] Maassen CBM, van Holten-Neelen JCPA, Balk F, Heijne den Bak-Glashouwer MJ, Leer RJ, Laman JD, et al. Strain dependent induction of cytokine profiles in the gut by orally administered Lactobacillus strains. Vaccine 2000;18(23):2613-23.

[35] Maassen CB, Boersma WJ, van Holten-Neelen C, Claassen E, Laman JD. Growth phase of orally administered Lactobacillus strains differentially affects IgG1//gG2a ratio for soluble antigens: implications for vaccine development. Vaccine 2003;21: 2751-7.

[36] Deibler GE, Martenson RE, Kies MW. Large scale preparation of myelin basic protein from central nervous tissue of several mammalian species. Prep Biochem 1972;2(2):139-65.

[37] Prakken BJ, van der Zee R, Anderton SM, van Kooten PJS, Kuis W, van Eden W. Peptide-induced nasal tolerance for a mycobacterial heat shock protein $60 \mathrm{~T}$ cell epitope in rats suppresses both adjuvant arthritis and nonmicrobially induced experimental arthritis. Proc Natl Acad Sci USA 1997;94(7):3284-9.
[38] Laman JD, Maassen CBM, Schellekens MM, Visser L, Kap M, de Jong E, et al. Therapy with antibodies against CD40L (CD154) and CD44-variant isoforms reduces experimental autoimmune encephalomyelitis induced by a proteolipid protein peptide. Mult Scler 1998;4(3):147-53.

[39] Shaw DM, Gaerthe B, Leer RJ, et al. Engineering the microflora to vaccinate the mucosa: serum immunoglobulin $G$ responses and activated draining cervical lymph nodes following mucosal application of tetanus toxin fragment $\mathrm{C}$-expressing lactobacilli. Immunology 2000;100(4):510-8.

[40] Zegers ND, Kluter E, van Der Stap H, et al. Expression of the protective antigen of Bacillus anthracis by Lactobacillus casei: towards the development of an oral vaccine against anthrax. Appl Microbiol 1999;87(2):309-14.

[41] Medaglini D, Oggioni MR, Pozzi G. Vaginal immunization with recombinant Gram-positive bacteria. Am J Reprod Immunol 1998;39(3):199-208.

[42] Kruger C, Hu Y, Pan Q, et al. In situ delivery of passive immunity by lactobacilli producing single-chain antibodies. Nat Biotechnol 2002;20(7):702-6.

[43] Kruisselbrink A, Heijne Den Bak-Glashouwer MJ, Havenith CE, Thole JE, Janssen R. Recombinant Lactobacillus plantarum inhibits house dust mite-specific T-cell responses. Clin Exp Immunol 2001;126(1):2-8.

[44] Bai XF, Li HL, Shi FD, et al. Complexities of applying nasal tolerance induction as a therapy for ongoing relapsing experimental autoimmune encephalomyelitis (EAE) in DA rats. Clin Exp Immunol 1998;111(1):205-10.

[45] Xiao BG, Link H. Mucosal tolerance: a two-edged sword to prevent and treat autoimmune diseases. Clin Immunol Immunopathol 1997;85(2):119-28.

[46] Genain CP, Abel K, Belmar N, et al. Late complications of immune deviation therapy in a nonhuman primate. Science 1996;274(5295):2054-7.

[47] Fairweather NF, Chatfield SN, Makoff AJ, Strugnell RA, Bester J, Maskell DJ, et al. Oral vaccination of mice against tetanus by use of a live attenuated Salmonella carrier. Infect Immun 1990;58(5):1323-6.

[48] Maassen CBM, van Holten JCPA, Balk F, Heijne den Bak-Glashouwer MJ, Leer R, Laman JD, et al. Orally administered Lactobacillus strains differentially affect the direction and efficacy of the immune response. Vet Q 1998;20(Suppl 3):S81-3.

[49] Javed NH, Gienapp IE, Cox KL, Whitacre CC. Exquisite peptide specificity of oral tolerance in experimental autoimmune encephacomyelitis. J Immunol 1995;155(3):1599-605.

[50] Lamont AG, Mowat AM, Parrott DM. Priming of systemic and local delayed-type hypersensitivity responses by feeding low doses of ovalbumin to mice. Immunology 1989;66(4):595-9.

[51] Hashim GA, Day ED, Fredane L, Intintola P, Carvalho E. Biological activity of region $65-102$ of the myelin basic protein. $\mathrm{J}$ Neurosci Res 1986;16(3):467-78.

[52] Fritz RB, Chou FC, Chou CH, Kibler RF. The immune response of Lewis rats to peptide $68-88$ of guinea pig myelin basic protein. II. B cell determinants. J Immunol 1979;123(4):1544-7.

[53] Cross ML. Microbes versus microbes: immune signals generated by probiotic lactobacilli and their role in protection against microbial pathogens. FEMS Immunol Med Microbiol 2002;34(4):245-53.

[54] Christensen HR, Frokiaer H, Pestka JJ. Lactobacilli differentially modulate expression of cytokines and maturation surface markers in murine dendritic cells. J Immunol 2002;168(1):171-8.

[55] Lutz MB, Schuler G. Immature, semi-mature and fully mature dendritic cells: which signals induce tolerance or immunity? Trends Immunol 2002;23(9):445-9. 